\title{
Lagrangian dispersion and heat transport in convective turbulence
}

\author{
Jörg Schumacher ${ }^{1,2}$ \\ ${ }^{1}$ Department of Mechanical Engineering, Technische Universität Ilmenau, D-98684 Ilmenau, Germany, \\ ${ }^{2}$ International Collaboration for Turbulence Research (ICTR)
}

(Dated: October 31, 2018)

\begin{abstract}
Lagrangian studies of the local temperature mixing and heat transport in turbulent RayleighBénard convection are presented, based on three-dimensional direct numerical simulations. Contrary to vertical pair distances, the temporal growth of lateral pair distances agrees with the Richardson law, but yields a smaller Richardson constant due to correlated pair motion in plumes. Our results thus imply that Richardson dispersion is also found in anisotropic turbulence. We find that extremely large vertical accelerations appear less frequently than lateral ones and are not connected with rising or falling thermal plumes. The height-dependent joint Lagrangian statistics of vertical acceleration and local heat transfer allows us to identify a zone which is dominated by thermal plume mixing.
\end{abstract}

PACS numbers: 47.55.pb,47.27.te

Introduction. The interplay of turbulent temperature and velocity fields in thermal convection is present in many systems reaching from astro- and geophysical flows [1, 2] to chemical engineering and indoor ventilation [3]. Almost all experimental and numerical studies on thermal convection have been carried out in the Eulerian frame of reference where the velocity and the temperature are analysed at fixed spatial positions and the mean turbulent heat transfer is measured with increasing precision for ever larger Rayleigh numbers [4, 5, 6]. Not much is however known about the local mechanisms of the transport of heat and momentum. Heat transport fluctuates locallly since it is arranged in coherent thermal plumes. They detach permanently from the thermal boundary layers, carry blobs of hotter or colder fluid into the bulk and cause an efficient temperature mixing. The complementary Lagrangian description in which turbulent fields are monitored along the tracks of fluid parcels [7, 8, 9, 10, 11, 12, 13, 14] could thus provide exactly the missing insights on the local mixing and transport mechanisms in convective turbulence. For example, the frequency and seasonal variability of thermal plumes affects the transport and concentration of phytoplankton in the upper ocean and has an impact on the carbon cycle [15, 16]. Vertically transported graupel particles or water droplets in deep-convective cumulus clouds determine how much precipitation falls out [17, 18].

In this Letter, we study the Lagrangian mixing of turbulent Rayleigh-Bénard convection in three-dimensional direct numerical simulations. Two aspects are in the focus of the present investigation. Firstly, we provide a detailed analysis of the acceleration and dispersion properties of Lagrangian tracers in convection. Caused by the interplay of buoyancy and gravity, Rayleigh-Bénard turbulence is inhomogeneous and anisotropic. Consequently, significant differences between the vertical and lateral transport properties are found. Secondly, we will take a "Lagrangian fingerprint" of thermal plumes. We therefore explore the statistics of local heat transfer and its relation to the local acceleration along the Lagrangian tracer tracks, all this as a function of height. This al-

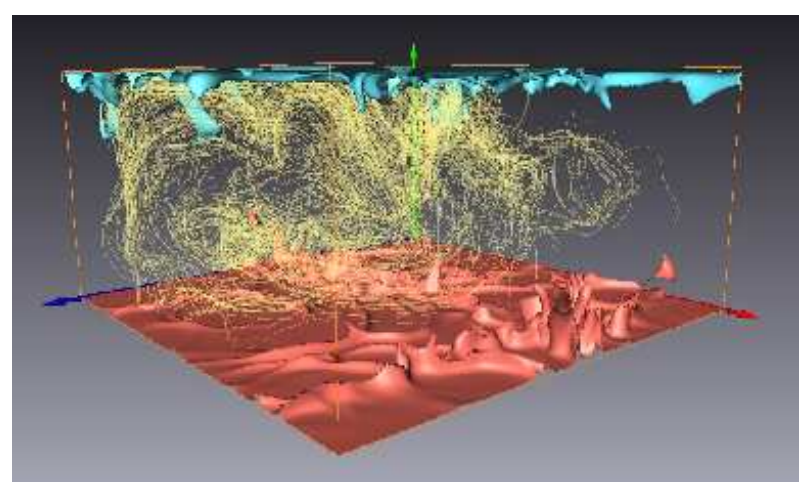

FIG. 1: (Color online) Instantaneous snapshot of two isosurfaces of the total temperature $T$ (top=cold, bottom $=$ hot). Thermal plumes which detach from both planes can be observed. Streamlines of the velocity field are also shown.

lows us to identify the regions in the convection cell which are dominated by the plumes and in which consequently enhanced vertical temperature mixing is present [4]. Our studies extend recent Eulerian investigations on the plume structure [19, 20] and an experiment with neutrally buoyant smart temperature sensors which was able to probe Lagrangian bulk properties in high-Rayleighnumber convection on scales down to 17 times the thermal boundary layer thickness [21].

Numerical simulations. The Boussinesq equations for an incompressible flow and the advection-diffusion equation for the temperature field, are solved by a standard pseudospectral method for the three-dimensional case. The equations are given by

$$
\begin{aligned}
\boldsymbol{\nabla} \cdot \boldsymbol{u} & =0 \\
\frac{\partial \boldsymbol{u}}{\partial t}+(\boldsymbol{u} \cdot \boldsymbol{\nabla}) \boldsymbol{u} & =-\nabla p+\nu \boldsymbol{\nabla}^{2} \boldsymbol{u}+\alpha g \theta \boldsymbol{e}_{z} \\
\frac{\partial \theta}{\partial t}+(\boldsymbol{u} \cdot \boldsymbol{\nabla}) \theta & =\kappa \boldsymbol{\nabla}^{2} \theta+u_{z} \frac{\Delta T}{H}
\end{aligned}
$$

Here, $\boldsymbol{u}$ is the turbulent velocity field, $p$ the pressure field and $\theta$ the temperature field. The system parameters 

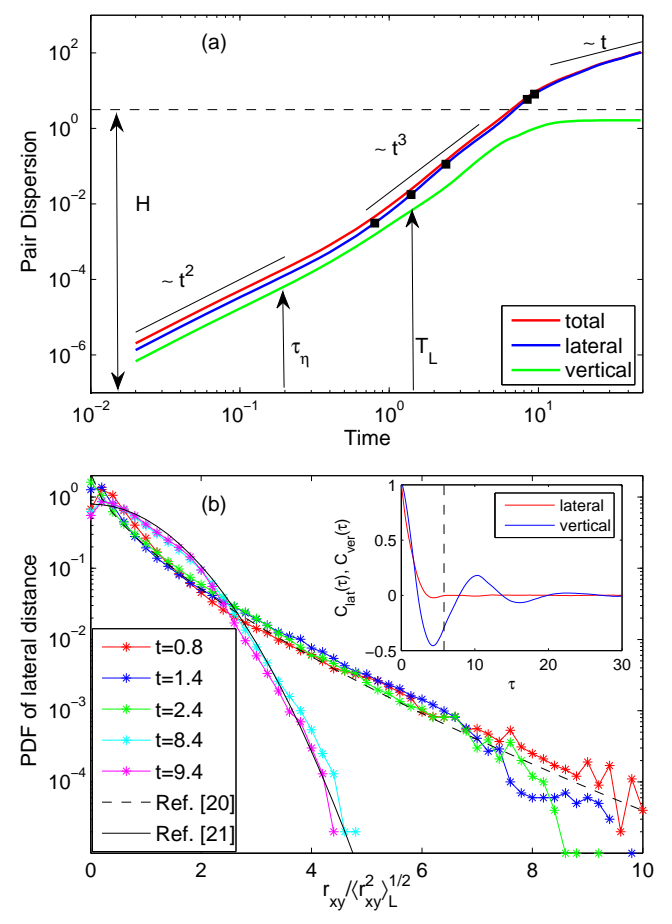

FIG. 2: (Color online) Particle pair dispersion in turbulent convection. (a): Pair dispersion $\left\langle[\boldsymbol{\Delta}(t)-\boldsymbol{\Delta}(0)]^{2}\right\rangle_{L}$ as a function of time $t$. The vector $\boldsymbol{\Delta}$ is the distance vector between both tracer positions within a pair. The vertical, lateral and total dispersion are compared. The height of the convection cell, the Kolmogorov time, $\tau_{\eta}$, the lateral Lagrangian integral time, $T_{L}$, are also indicated. (b): Probability density function (PDF) of the lateral dispersion taken at four times, see symbols in (a). For comparison, we include the Gaussian prediction by Batchelor [23] and the Richardson prediction [22] (with different fit factors). The inset shows the Lagrangian velocity autocorrelation functions for the lateral, $C_{\text {lat }}(\tau)$ and vertical , $C_{\text {ver }}(\tau)$ components. The vertical dashed line corresponds with the travel time $H / u_{z, r m s}$.

are: gravity acceleration $g$, kinematic viscosity $\nu$, thermal diffusivity $\kappa$, vertical temperature gradient $\Delta T / H$, and thermal expansion coefficient $\alpha$. The total temperature field $T$ is decomposed into a linear background profile and a fluctuating field $\theta$

$$
T(\boldsymbol{x}, t)=-\frac{\Delta T}{H}(z-H / 2)+\theta(\boldsymbol{x}, t) .
$$

Since $T= \pm \Delta T / 2$ at boundaries $z=0$ and $z=$ $H(=\pi)$, the boundary condition $\theta=0$ follows. Here, $\Delta T>0$. The dimensionless control parameters are the Prandtl number $\operatorname{Pr}=\nu / \kappa=0.7$, the Rayleigh number $R a=\alpha g H^{3} \Delta T /(\nu \kappa)=1.2 \times 10^{8}$, and the aspect ratio $\Gamma=L / H=2$ with $L=L_{x}=L_{y}=2 \pi$. Figure 1 illustrates a typical snapshot of the flow and temperature fields. In the vertical direction $z$, free-slip boundary conditions are used. Laterally in $x$ and $y$, periodic boundary conditions are applied. The simulation box is resolved by $512 \times 512 \times 257$ grid points which results in a spectral

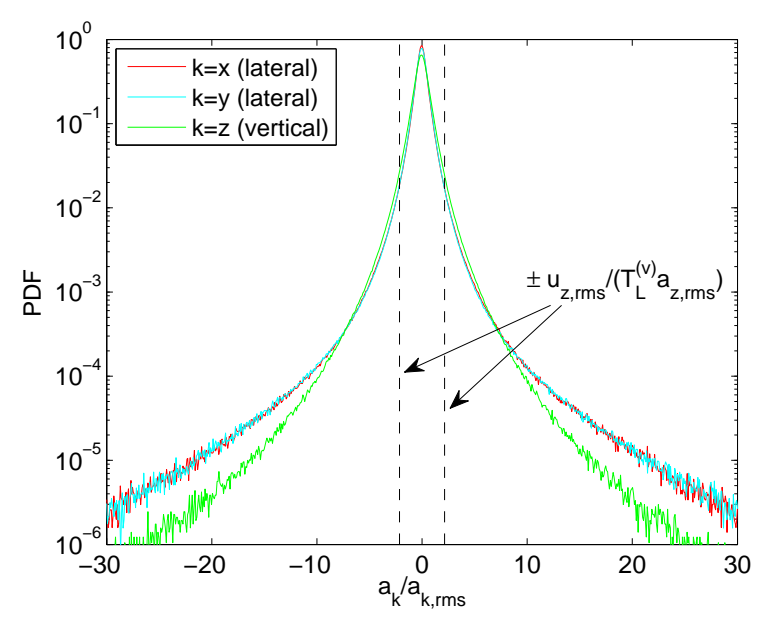

FIG. 3: (Color online) Probability density function (PDF) of the acceleration vector components. Components are normalized by their corresponding root-mean-square values (rms). The skewness of all three distributions vaires between -0.2 and -0.09 . The dashed vertical lines mark the characteristic acceleration $u_{z, r m s} / T_{L}^{(v)}$.

resolution of $k_{\max } \eta_{K}=2.2$. The Taylor microscale The Reynolds number is $R_{\lambda}=135$ and the Nusselt number $N u=58.05 \pm 0.98$. Time-stepping is done by a secondorder predictor-corrector scheme. We track $N=10^{6} \mathrm{La}-$ grangian tracers simultaneously with the flow. Initially, they are arranged in pairs with a separation of 1.5 grid cell widths. Intergrid velocities are obtained by trilinear interpolation.

Particle dispersion. Figure 2a reports our findings on the pair dispersion which is given by $R^{2}(t)=\langle[\boldsymbol{\Delta}(t)-$ $\left.\boldsymbol{\Delta}(0)]^{2}\right\rangle_{L}$. The statistics is taken over $N / 2$ tracer pairs and denoted as $\langle\cdot\rangle_{L}$. Each pair is described by the particle distance vector $\boldsymbol{\Delta}=\boldsymbol{x}_{2}-\boldsymbol{x}_{1}$. Interestingly, after the initial ballistic growth with $t^{2}$ a crossover to a Richardson-like dispersion with $R^{2}(t)=C_{2}\langle\epsilon\rangle t^{3}$ is observed, similar to isotropic fluid turbulence [7, 11, 22]. Here, $\langle\epsilon\rangle$ is the mean energy dissipation rate. In contrast to the isotropic case, the Richardson constant is however reduced by two third and is given by $C_{2}=0.16$ in the present study [7, 11]. Recall, that the turbulence is inhomogeneous with respect to the vertical direction. Therefore, we decompose the distance vector into a lateral and vertical part, $\boldsymbol{\Delta}=\boldsymbol{r}_{x y}+r_{z} \boldsymbol{e}_{z}$. While the vertical distances cannot grow beyond the cell height $H$, the lateral one takes over and makes up the whole large-time growth of $R^{2}(t)$ including the Richardson scaling. Eventually, the lateral pair motion becomes decorrelated and changes to the diffusive limit with $R^{2}(t) \sim t$ (see Fig. 2a).

In Fig. 2a, the viscous Kolmogorov time $\tau_{\eta}=\sqrt{\nu /\langle\epsilon\rangle}$ and the lateral Lagrangian integral time scale $T_{L}$ are given. Both are separated by less than an order of magnitude for the Reynolds number accessible here. Furthermore, the $t^{3}$-regime extends beyond $T_{L}$. Figure $2 \mathrm{~b}$ 
shows the probability density function (PDF) of the lateral distance at five different times. The PDFs collapse to a Richardson-like shape and clearly deviate from the Gaussian limit for times around $T_{L}$. They cross over to Gaussian shape for larger times. A Gaussian distribution, as predicted by Batchelor for the Lagrangian inertial range, is not supported by the present study [23].

The inset of Fig. $2 \mathrm{~b}$ unravels another specifics in convection. The Lagrangian velocity autocorrelation functions of the vertical and lateral components, $C_{v e r}(\tau)$ and $C_{\text {lat }}(\tau)$, which are calculated along the tracer tracks are shown. The strong anticorrelation of the vertical part is due to the constraints in vertical motion, e.g. when the tracers (and the plumes) hit the top and bottom planes. The pronounced minimum in the vertical correlation is close to a characteristic vertical travel time $\sim H / u_{z, r m s}$, where $u_{z, r m s}=\left\langle u_{z}^{2}\right\rangle^{1 / 2}$. The integration of $C_{\text {ver }}(\tau)$ with respect to time results in a vertical Lagrangian integral time which is $T_{L}^{(v)} \approx T_{L} / 6$. While the single particle motion decorrelates on a shorter time scale as in isotropic turbulence, the lateral pair motion seems to be stronger correlated. The latter is due to tracer pair motion in plumes. This circumstence rationalizes the smaller Richardson constant $C_{2}$ and why $R^{2}(t) \sim t^{3}$ seems to continue for times $t>T_{L}$. Our studies demonstrate, that Richardson dispersion can also be found in anisotropic turbulence. They could thus shed light on why Richardson-like dispersion is frequently observed, but the constant $C_{2}$ strongly scatters around the analytically predicted value.

Lagrangian plume detection. The results on the particle pair dispersion suggest to take a closer look to the vertical accelerations and how their amplitudes are correlated with the thermal plumes. In Fig. 3, we show first the PDFs of all three acceleration components. As expected, the PDFs of both lateral components, $a_{x}$ and $a_{y}$, collapse onto each other. Surprisingly, they yield fatter tails compared to the remaining vertical acceleration component. The characteristic amplitude for the latter can be estimated by $\left|a_{z}\right| \sim u_{z, r m s} / T_{L}^{(v)}$ which is indicated in Fig. 3. The largest vertical acceleration amplitudes result then to about 10 times this estimate. The overall symmetric and stretched exponential shape reveals the strong intermittency of Lagrangian motion as in isotropic fluid turbulence [12, 14]. The differences in lateral and vertical accelerations suggest that the rise of buoyant plumes is less gradual than the lateral vortical motion.

It is known that rising or falling plumes can be identified by a positive product of vertical velocity and temperature fluctuation, $u_{z} T^{\prime}>0$ where $T^{\prime}(\boldsymbol{x}, t)=$ $T(\boldsymbol{x}, t)-\langle T\rangle_{A}(z)$. Here, $\langle\cdot\rangle_{A}$ denotes ensemble averages at fixed height $z$. The product $u_{z} T^{\prime}$ is directly related to the (Lagrangian) Nusselt number [21] which is given by $N u_{L}=1+H /(\kappa \Delta T) u_{z} T^{\prime}$. Figure 4c shows how probable the positive and negative amplitudes of $u_{z} T^{\prime}$ as a function of height $z$ are. We see, that the distribution is always asymmetric with respect to the axis $u_{z} T^{\prime}=0$.
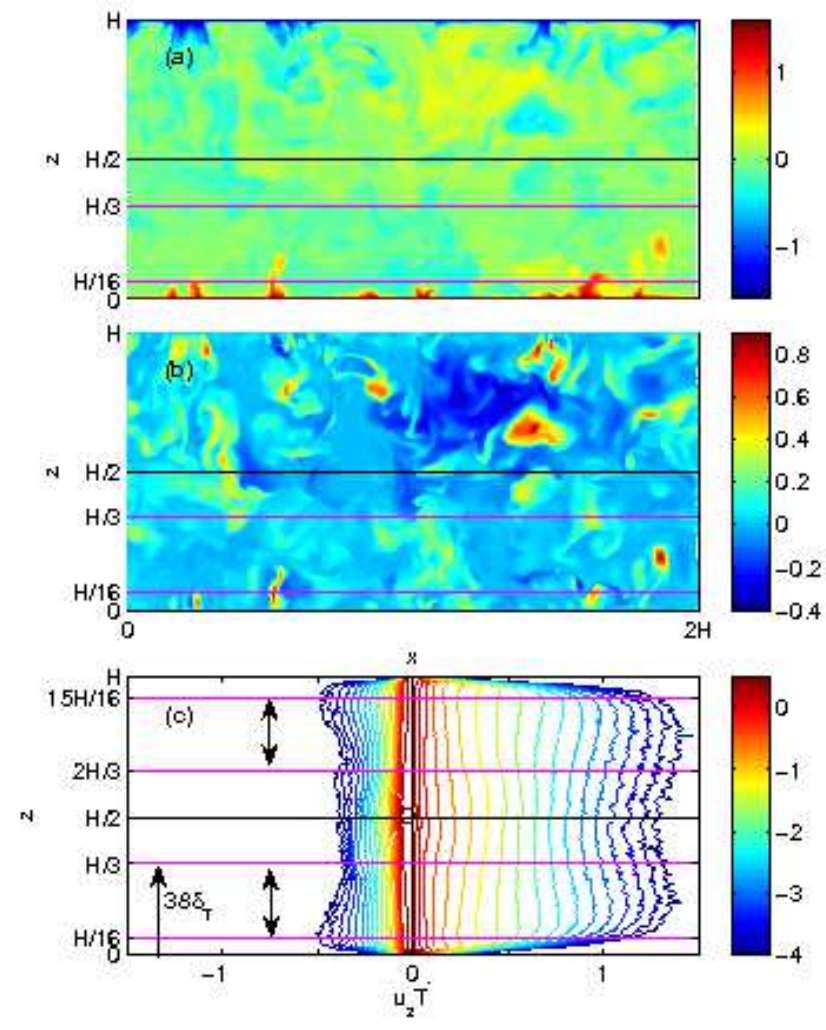

FIG. 4: (Color online) Lagrangian heat flux as a function of height. (a) Vertical slice cut through the instantaneous temperature field $T$. (b) Corresponding product field $u_{z} T^{\prime}$. (c) Lagrangian probability density function (PDF) of $u_{z} T^{\prime}$ as a function of vertical tracer position $z$. The height $z=$ $H / 3$ corresponds with 38 times the thermal boundary layer thickness $\delta_{T}$. The regions between both thick horizontal lines at $H / 3$ and $H / 16$ as well as $2 H / 3$ and $15 H / 16$ are considered as the mixing zones. Color legend is here in decadic logarithm.

This reflects the net transfer of heat from the bottom to the top plane. The broad support of the PDF is due to the fluctuations of the local heat transfer. The joint PDF shows two features. Firstly, the support of the PDF for $u_{z} T^{\prime}>0$ is broadest at about 18 times the thermal boundary layer thickness $\delta_{T}$ (i.e. $\left.z \approx H / 6\right)$. Secondly, the support of the PDF for $u_{z} T^{\prime}<0$ peaks at $H / 16$ and decreases to about $H / 3$. In order to shed more light on the structural features which are associated with this statisitics, we show in Fig. 4 the temperature $T$ (a) and the product $u_{z} T^{\prime}$ (b). Clearly, thermal plumes are found were $u_{z} T^{\prime}>0$. However, one observes also larger $u_{z} T^{\prime}<0$ in the vicinity of plumes. Due to the incompressibility of the flow, strong upwellings are accompanied by neighboring downwellings which reflects in the statistical distribution in the bottom panel of Fig. 4. By combining both aspects, we conclude that the heat transport and mixing are dominated by thermal plumes in the zone between $H / 16 \lesssim z \lesssim H / 3$. Such plume mixing re- 


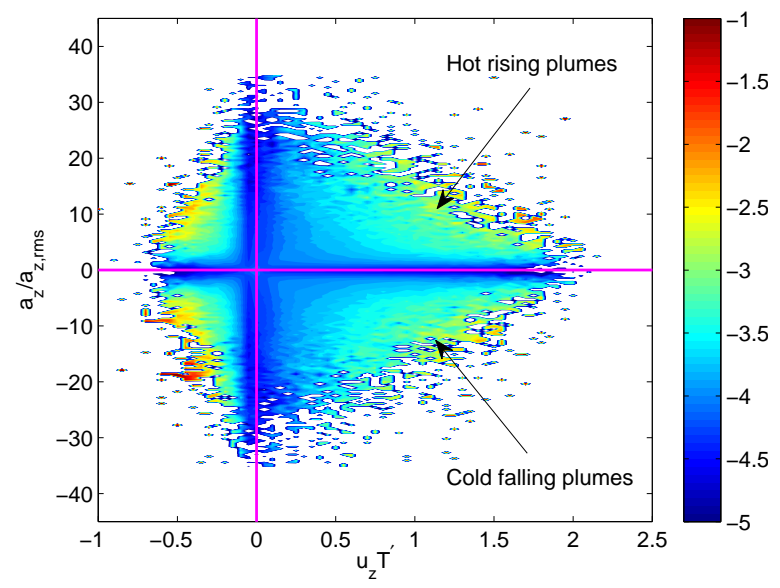

FIG. 5: (Color online) Joint Lagrangian PDF of vertical acceleration component $a_{z} / a_{z, r m s}$ and the product $u_{z} T^{\prime}$. Color coding is again in decadic logarithmic units as indicated by the color bar. The regions for rising hot plumes and falling cold plumes are indicated by arrows.

gion has been suggested by Castaing et al. [4] and was determined in an Eulerian analysis 24]. Compared to [24], the present mixing zone is smaller in extension, but again significantly larger as the thermal boundary layer thickness. This explains why the smart sensor experiment by Gasteuil et al. 21] could detect successfully the large fluctuations of the heat transfer despite the large probe diameter.

Finally, the combination of the results on acceleration and local heat transfer result in the joint statistics which is shown in Fig. 5. In order to highlight the statistical dependence of both fields, we normalize the joint PDF by the single quantity PDFs, $\Pi\left(a_{z}, u_{z} T^{\prime}\right)=$
$P\left(a_{z}, u_{z} T^{\prime}\right) /\left[P\left(a_{z}\right) P\left(u_{z} T^{\prime}\right)\right]$. The pronounced asymmetry with respect to the axis $u_{z} T^{\prime}=0$ reflects again the net transfer of heat, similar to Fig.4. It can be seen that larger accelerations are associated with rising and falling plumes as well as with resulting down- and upwellings, respectively. The latter are found in the corresponding diagonal quadrant of the plane. Maxima of $\Pi\left(a_{z}, u_{z} T^{\prime}\right)$ are detected in the outer regions of the support, but not for the strongest acceleration. The figure illustrates thus also which fraction of the tail of the acceleration PDF is most probably associated with the plume motion.

Summary. Due to vertical buoyancy driving of convective turbulence, Lagrangian tracer motion is found to be strongly anisotropic in contrast to classical fluid turbulence [7, 11]. On the one hand, the vertical Lagrangian transport is found significantly shorter-in-time correlated than the lateral one. On the other hand, rising thermal plumes seem to enforce a more coherent lateral motion which results in a smaller Richardson constant for the lateral Richardson pair dispersion which has been detected here in an anisotropic flow. Vertical accelerations are diminished compared to lateral ones and their largest amplitudes are not connected with rising and falling plumes. Based on the Lagrangian monitoring of heat transfer, the plume mixing zone has been identified complementary to previous Eulerian analysis. It is believed, that the presented features can be carried over qualitatively to the case of no-slip boundaries. This particular investigation and trends with increasing Rayleigh number are part of our future work.

Acknowledgements. The author acknowledges support by the Deutsche Forschungsgemeinschaft (DFG) under grant SCHU 1410/2. The direct numerical simulations have been carried out at the IBM-p690 cluster JUMP and the IBM Blue Gene/L JUBL at the Jülich Supercomputing Centre (Germany) under grant HIL02.
[1] F. Cattaneo, T. Emonet, and N. Weiss, Astrophys. J. 588, 1183 (2003).

[2] B. Stevens, Annu. Rev. Earth Planet. Sci. 33, 605 (2005).

[3] P. F. Linden, Annu. Rev. Fluid Mech. 31, 201 (1999).

[4] B. Castaing, G. Gunarante, F. Heslot, L. P. Kadanoff, A. Libchaber, S. Thomae, X.-Z. Wu, S. Zaleski, and G. Zanetti, J. Fluid Mech. 204, 1 (1989).

[5] J. J. Niemela, L. Skrebk, K. R. Sreenivasan, and R. J. Donelly, Nature 404837 (2000).

[6] D. Funfschilling, E. Brown, A. Nikolaenko, and G. Ahlers, J. Fluid Mech. 536, 145 (2005).

[7] S. Ott and J. Mann, J. Fluid Mech. 422, 207 (2000).

[8] A. La Porta, G. A. Voth, A. M. Crawford, J. Alexander, and E. Bodenschatz, Nature 409, 1017 (2001).

[9] N. Mordant, P. Metz, O. Michel und J.-F. Pinton, Phys. Rev. Lett. 87, 214501 (2001).

[10] P. K. Yeung, Annu. Rev. Fluid Mech. 34, 115 (2002).

[11] G. Boffetta and I. M. Sokolov, Phys. Rev. Lett. 88, 094501 (2002).

[12] L. Biferale, G. Boffetta, A. Celani, B. J. Devenish, A.
Lanotte, and F. Toschi, Phys. Rev. Lett. 93, 064502 (2004).

[13] M. Bourgoin, N. T. Ouelette, H. Xu, J. Berg, and E. Bodenschatz, Science 311, 835 (2006).

[14] H. Homann, R. Grauer, A. Busse, and W. C. Müller, J. Plasma Phys. 73, 821 (2007).

[15] A. Körtzinger, J. Schimanski, U. Send, and D. Wallace, Science 306, 1337 (2004).

[16] S. C. Riser and K. S. Johnson, Nature 451, 323 (2008).

[17] A. M. Blyth, J. Appl. Meteorology 32, 626 (1993).

[18] R. A. Shaw, Annu. Rev. Fluid Mech. 35, 183 (2003).

[19] O. Shishkina and C. Wagner, Phys. Fluids 19, 085107 (2007).

[20] S.-Q. Zhou, C. Sun, and K.-Q. Xia, Phys. Rev. Lett. 98, 074501 (2007).

[21] Y. Gasteuil, W. L. Shew, M. Gilbert, F. Chillá, B. Castaing, and J.-F. Pinton, Phys. Rev. Lett. 99, 234302 (2007).

[22] L. F. Richardson, Proc. Roy. Soc. London Ser. A 110, 709 (1926). 
[23] G. K. Batchelor, Q. J. R. Meteorol. Soc. 76, 133 (1950).

[24] S.-Q. Zhou and K.-Q. Xia, Phys. Rev. Lett. 89, 184502

(2002). 